\title{
Microfluidic-based measurements of cytochrome P450 enzyme activity of primary mammalian hepatocytes
}

\author{
Keith Anderson, ${ }^{a}$ Jonathan M. Cooper, ${ }^{b}$ Stephen J. Haswell, ${ }^{c}$ Damian Marshall, ${ }^{a}$ Huabing Yin ${ }^{b}$ \\ and Xunli Zhang*cd
}

\author{
Received 24th January 2010, Accepted 19th March 2010 \\ First published as an Advance Article on the web 17th April 2010 \\ DOI: 10.1039/c0an00031k
}

A microfluidic-based system was developed for the in situ monitoring of the 7-ethoxyresorufin O-dealkylation (EROD) activity of primary rat hepatocytes by measuring the fluorescent intensity of both cells and their surrounding media. The microfluidic chip was designed to allow the cell suspension and test reagent to be introduced in a layer-by-layer flow format, thereby resulting in a short mixing time by diffusion. A good linear relationship was obtained between the resorufin concentration up to $30 \mu \mathrm{M}$ and fluorescent intensity over the chip's circular chamber area. The EROD activity was determined with 3-methylcholanthrene (3-MC)-induced hepatocytes. The inhibition effect of $\alpha$-naphthoflavone was also examined on EROD activity resulting in an $\mathrm{IC}_{50}$ value of $12.98 \mu \mathrm{M}$.

\section{Introduction}

Hepatic cytochrome P450 (CYP450) is a family of variant enzymes that catalyses the oxidative metabolism of a wide variety of exogenous chemicals including drugs, carcinogens, toxins and endogenous compounds such as steroids, fatty acids and prostaglandins. ${ }^{\mathbf{1 , 2}}$ The quantitative determination of CYP450 enzymatic activity in mammalian hepatocytes is a commonly used method for drug screening and detecting general toxicity where CYP activity can be induced or inhibited by specific test compounds. ${ }^{3-5}$ One of the commonly used assays for CYP450 enzymatic activity is 7-ethoxyresorufin O-dealkylation (EROD), which is based on the conversion of 7-ethoxyresorufin (7-ER) to resorufin, a reaction catalysed by CYP4501A enzymes (eqn (1)). ${ }^{6-8}$ Since the product is fluorescent, the kinetics of the reaction can be quantified by the measurement of fluorescence intensity during the reaction, which is directly related to CYP4501A enzymatic activity.

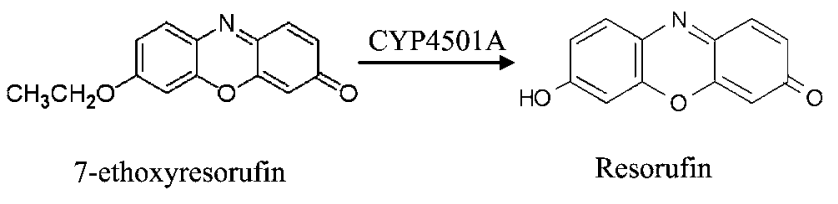

The determination of EROD activity is typically carried out in a multi-well (e.g., 96-well) plate using a fluorescence reader to record the signal associated with resorufin. ${ }^{5,7}$ Although the

${ }^{a}$ LGC Limited, Queens Road, Teddington, Middlesex, TW11 OLY, UK ${ }^{b}$ Department of Electronics and Electrical Engineering, University of Glasgow, Glasgow, G12 8QQ, UK

'Department of Chemistry, The University of Hull, Hull, HU6 7RX, UK

${ }^{d}$ Bioengineering Group, School of Engineering Sciences, University of Southampton, Southampton, SO17 1BJ, UK. E-mail: XL.Zhang@soton. ac.uk; Fax: +44 (0) 238059 3016; Tel: +44 (0) 2380595099 commonly used multi-well system can provide useful information on cell behaviour, function and genotype, based on the average measurements of cell populations, it is incapable of measuring the kinetics of enzyme reactions occurring within individual cells. In the case of EROD process, it involves both reaction (eqn (1)) and subsequent mass transport steps where fluorescent resorufin produced transfers by diffusion across the cell membrane towards the outside, and a closer examination of the reaction step is important for understanding the enzymatic reaction kinetics and mechanism. However, conventional methods of cell observation are unable to distinguish between these two steps.

In recent years, there has been an increasing awareness of the importance of cell heterogeneity in clinical diagnostics, which has been reflected in the shift from studying large cell populations (to obtain average cell responses) to single cell measurements. ${ }^{9-11}$ The development of single cell-based analysis not only provides complementary information on a cell population but can also help reveal the actual functional interaction of biomolecules at both a cellular and tissue level. ${ }^{12,10}$

Over the past two decades, significant developments in the field of miniaturised systems, so-called microfluidics or lab-on-a-chip technologies, have seen the methodology influence diverse areas of applications relating to analytical chemistry, biochemistry, clinical diagnosis, medical chemistry and industrial chemistry. ${ }^{13,14}$ It has been demonstrated that such microsystems represent advantages over conventional bench systems in terms of speed, performance, reduced sample/solvent quantity, integration and automation. Of particular relevance to cell analysis is the small length scale used in which fluidics are restricted to diffusive mixing under laminar flow, thereby enabling precise fluidic control to create a biomimetic cell microenvironment. The microfluidic control also provides unique opportunities for delivering and locating single cells within microchips with an appropriate micro-geometry design. In addition, coupling with microscope-based imaging techniques allows the detection and measurement of single cell and subcellular levels in situ. ${ }^{15}$ 
Consequently, a wide range of microfluidic-based methodologies has been developed for cell manipulation, culture and analysis including hepatocytes. ${ }^{4,16}$

The aim of this feasibility study is to develop a microfluidicbased system that enables the in situ monitoring of EROD activity using primary rat hepatocytes by measuring the fluorescent intensity of both single cells and the surrounding media. The inhibition of EROD activity will be examined using $\alpha$-naphthoflavone $(\alpha-N F)$ as a CYP1A inhibitor over a range of concentrations $(0-40 \mu \mathrm{M})$.

\section{Materials and methods}

\subsection{Microfluidic chip fabrication}

The microfluidic device was produced according to published procedures with minor adaptations. ${ }^{17,18}$ Briefly, the channel network was fabricated using a photolithographic fabrication method. The channel network was first designed using AutoCAD drawing software (Autodesk, Farnborough, UK). A film negative of the desired fluidic network was then prepared by a commercial photo mask manufacturer (J.D. Photo Tools, Oldham, UK) to form the optical mask. B-270 glass photolithographic plates (thickness of $3 \mathrm{~mm}$ ) coated with a thin chromium metal mask layer plus an upper layer of positive photoresist, supplied by Telic (Telic Company, Valencia, USA), were used for channel network fabrication. With UV exposure, the pattern of interconnecting channels was transferred from the optical mask to the photoresist layer, which was then developed and removed together with the chromium layer to reveal the channel areas of glass to be etched. The channels were etched using a mixture of $1 \%(\mathrm{w} / \mathrm{w}) \mathrm{HF}$ and $5 \%(\mathrm{w} / \mathrm{w}) \mathrm{NH}_{4} \mathrm{~F}$ in water at $65^{\circ} \mathrm{C}$.

The fabricated microchannel network consisted of a circular chamber (for cell holding and monitoring) with two inlet channels and one outlet channel (Fig. 1a). To create two layers of sheet flow in the main inlet channel and the chamber, the inlet channels were etched separately on two glass plates, which were then bonded face-to-face. Channels on each glass plate were etched to a depth of $35 \mu \mathrm{m}$, resulting in a total depth of $70 \mu \mathrm{m}$ for the main channel and the chamber. The width for all the channels was $150 \mu \mathrm{m}$, and the circular chamber had a diameter of $900 \mu \mathrm{m}$, providing a working volume of $0.0445 \mu \mathrm{l}$. The main purpose of using lay-by-layer sheet flow instead of side-by-side flow was to reduce the diffusion distance in order to shorten the diffusive mixing time whilst providing a relatively large field of view.

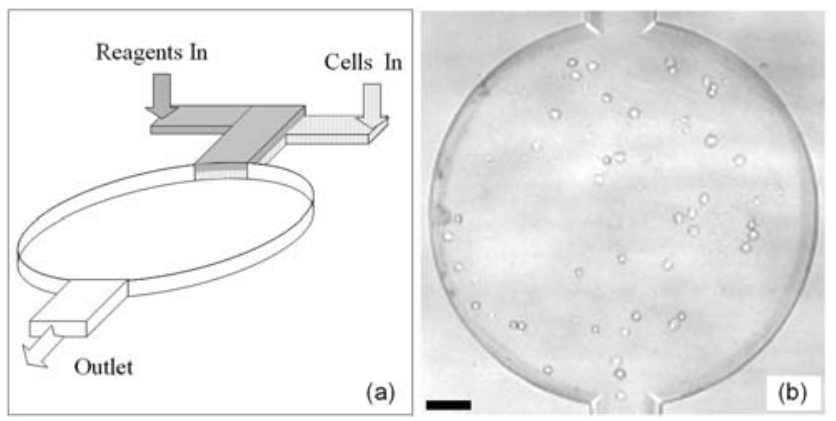

Fig. 1 Schematic of chip design (a) and hepatocytes in suspension loaded onto the chip in the circular chamber (b). Scale bar: $100 \mu \mathrm{m}$.
Three holes (dia. $1.5 \mathrm{~mm}$ ) were drilled through the upper glass plate to link the ends of the channels with tubing. The upper plate was then aligned with the channel geometry on the base plate and thermally bonded by placing it in a muffle furnace set at $570{ }^{\circ} \mathrm{C}$ for $3 \mathrm{~h}$. A $90 \mathrm{~g}$ block of stainless steel was placed onto the upper plate to assist bonding.

\subsection{Cell culture and assay reagents}

Preserved rat hepatocytes were supplied by Abcellute (Abcellute Ltd, Cardiff, UK) and maintained at a temperature of $10{ }^{\circ} \mathrm{C}$ during transportation. Both 3-methylcholanthrene (3-MC)induced $(72 \mathrm{~h})$ hepatocytes and controls were supplied. The preserved hepatocytes were then reactivated with the reactivation medium and wash medium, which were supplied with the cells, using the Abcellute protocol ABC-REC-S. The concentration of cells used for loading the chip during the experiment was in the range of $1 \times 10^{6}$ cells $\mathrm{ml}^{-1}$. The viability of hepatocytes was assessed prior to introduction on the chip using the trypan blue exclusion method with a haemocytometer.

The buffer solution was prepared by adding one pot of KHB (Krebs-Heinslett buffer) powder to 11 of distilled water in a volumetric flask supplemented by amikacin $\left(84 \mu \mathrm{g} \mathrm{ml}{ }^{-1}\right)$,

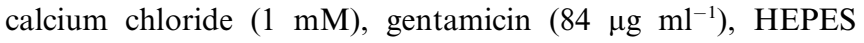
(20 mM), heptanoic acid $(4.2 \mu \mathrm{M})$ and sodium bicarbonate (28.5 mM), and then adjusted to $\mathrm{pH} 7.4$ with either $1 \mathrm{M} \mathrm{NaOH}$ or $1 \mathrm{M} \mathrm{HCl}$. Other chemicals were used as received, including 7-ER, resorufin, 3,3'-methylenebis(4-hydroxycoumarin) (or dicumarol), $\alpha-\mathrm{NF}$, acetonitrile and dimethyl sulfoxide (DMSO). All these materials were supplied by Sigma-Aldrich (Dorset, UK). To make the test reagent, dicumarol was first dissolved in DMSO and then diluted, together with 7-ER, in buffer to the desired concentration. The blank was DMSO only (at the equivalent concentration) added to the buffer.

\subsection{Instrumentation and image analysis}

An Axiovert S100 inverted microscope (Carl Zeiss, UK) using both transmission and fluorescent optics coupled with a monochrome CCD digital camera (C4742-95-12NRB, Hamamatsu Photonics, UK) was used to obtain both conventional micrographs and digital videos. AQM Hamamatsu ORCA I software (Kinetic Imaging, Nottingham, UK) was used for image acquisition and analysis. By selecting areas (e.g., cells or whole chamber) in successive video images a light intensity profile versus time was obtained. A Chroma green filter set (P/N 11002, Chroma Technology Corp, Rockingham, USA) was selected, which consists of an Exciter D546/10, an Emitter E590LP and a beam splitter Dichroic 565DCLP.

Two KDS 200 syringe pumps (KD Scientific Inc., Holliston, USA) were used to deliver cells in suspension and test reagents. ETFE (ethylene tetrafluoroethylene) polymer tubing with an inner diameter of $250 \mu \mathrm{m}$ and outer diameter of $1 / 16^{\prime \prime}(\mathrm{P} / \mathrm{N} 1529)$, on/off valves (P/N P-782) and appropriate fittings and connectors, all obtained from Upchurch (Upchurch Scientific Inc., Oak Harbor, USA), were used for plumbing to link the chip and syringes.

To maintain the chip temperature during measurements, a heated microscope stage (Model 14472, World Precision 
Instruments Ltd, Stevenage, UK) with an aperture of $6 \mathrm{~mm}$ in diameter was used and the temperature was controlled in the range of $37 \pm 0.5^{\circ} \mathrm{C}$. When the cells were loaded onto the chip, the measurement of EROD activity was carried out by taking a series of digital images as a video clip covering the whole circular chamber area of the chip under the fluorescent conditions. The scan rate of the video was set to be one frame every two minutes with an exposure time of $20 \mathrm{~ms}$. To minimise the photo bleaching effect, a shutter was inserted into the light path which was open (for 2 s) only when taking each image frame. All fluorescent images were taken under the same conditions for comparison and data analysis.

\section{Results and discussion}

\subsection{Cell loading}

Hepatocytes in suspension and the test reagent were introduced into the chip at a flow rate of $15 \mu \mathrm{min}^{-1}$ via the two inlet channels, in a continuous flow, layer-by-layer format (Fig. 1a). The test reagent was composed of $40 \mu \mathrm{M} 7$-ER and $80 \mu \mathrm{M}$ dicumarol in buffer, where dicumarol was added to prevent the disappearance of resorufin fluorescence since the product resorufin (eqn (1)) can be further reduced through metabolism by cytosolic oxidoreductases. ${ }^{7,19}$ Once a steady flow had been established, the on/off valves on all three inlet and outlet tubes were closed simultaneously, ${ }^{17}$ and the measurement process started. Fig. 1b shows the hepatocytes retained in the chip chamber in bright-field view after loading.

It can be seen from Fig. 1 that the delivery procedure allowed hepatocytes to be distributed reasonably evenly on the bottom of the chamber, whereas the test reagent solution was placed in the top layer above the cell/media. When the flow was stopped and all the valves were closed, cells in the chamber tended to settle down and attach to the bottom surface within about $10 \mathrm{~s}$, and diffusive mixing occurred based on Fick's law, ${ }^{20}$ which defines the relationship between the distance travelled, $d$, by a molecule and the time, $t$ :

$$
d=\sqrt{2 D t}
$$

where $D$ is the diffusion coefficient. Using this equation, the time needed for a resorufin molecule to diffuse across a distance of $70 \mu \mathrm{m}$, i.e., the chamber depth, is estimated to be $5.1 \mathrm{~s}$, based on the diffusion coefficient value of $4.80 \times 10^{-10} \mathrm{~m}^{2} \mathrm{~s}^{-1}$ as determined by Schilling et al. ${ }^{21}$ Compared to the typical measurement time of $20 \mathrm{~min}$ this time for diffusion was not expected to cause significant errors for kinetic measurements.

It was also observed that the hepatocytes were distributed as an attached monolayer within the $70 \mu \mathrm{m}$ deep chamber where the size of the hepatocytes was in the range of 10-20 $\mu \mathrm{m}$ in diameter. This monolayer distribution clearly simplified the process of image analysis and made it possible to select either a single cell or the surrounding media (outside the cell) for further analysis based on the fluorescent intensity. In addition, the cell viability assessment before and after passing through the chip without reaction over a given time period was not significantly different between the samples on the chip and in the batch micro-well plate.

\subsection{Single cell monitoring}

Upon the reaction taking place an increase in fluorescent intensity from the resorufin was observed for almost all hepatocytes. Fig. 2 shows the plot of fluorescent intensity of 12 individual cells randomly selected as a function of time (the fluorescent curves have been normalised for comparison). It was found that the fluorescent intensity for all cells showed a similar profile with some variations; fluorescent intensity increased approximately linearly for the first 10-12 min, reaching a maximum and then followed by a relatively stable period (and a gradual decline for some cells).

The fluorescent intensity of most cells (about nine out of 12) fell in a standard deviation of $\pm 15 \%$ compared with the average. This intensity difference was probably because of the variation in cell size. By examining the cell morphology (Fig. 1b), it was found that the cell size distribution for the majority of cells (about $80 \%$ ) was within the range of $\pm 20 \%$ compared with the average, which corresponds closely to the maxima for the cells' fluorescent intensities; the highest fluorescent intensity corresponds to the largest cell size and the lowest fluorescent intensity to the smallest cell size, although the variation in activity for individual cells cannot be ruled out.

However, the variation in the time needed for each single cell to reach the maximum fluorescence was indicative of alterations in reaction rates among individual cells. This method, therefore, can provide a useful tool for the measurement of the kinetics of enzyme reactions occurring within individual cells. It was also shown that the chip-based measurement required a remarkably low number of cells, which can be a major advantage when screening limited amounts of cell samples. ${ }^{22}$

\subsection{In situ measurement of EROD activity}

As discussed above, the measurement of EROD activity is based on the conversion of 7-ER (which is delivered with the test reagent) to fluorescent resorufin. ${ }^{23,24}$ This reaction was catalysed by cytochrome CYP1A, the expression of which was induced by 3-MC. Since the biocatalytic conversion of 7-ER to resorufin occurred inside hepatocytes, temporarily, the increase in fluorescent intensity started within hepatocytes and was followed by the fluorescent intensity increase in the surrounding media as

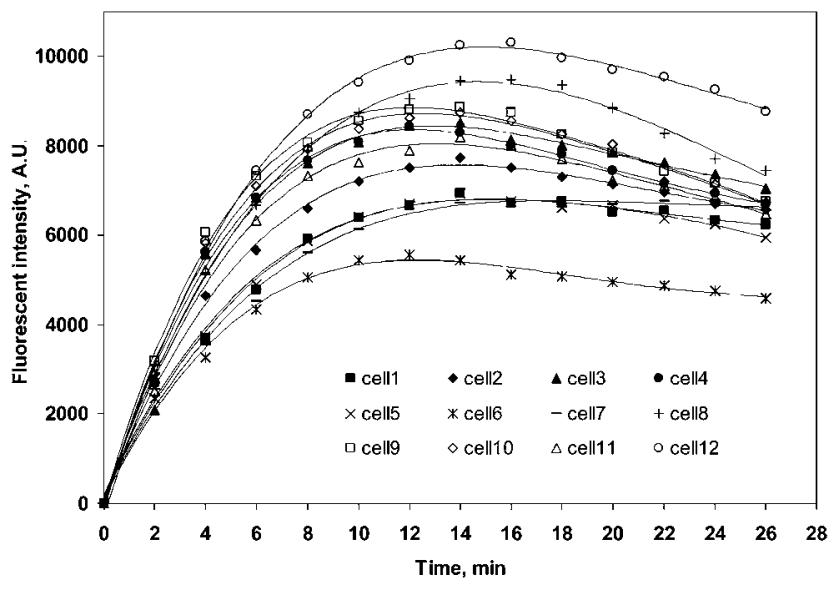

Fig. 2 Fluorescent intensity of single cells $(n=12) v s$. time. 
resorufin diffused outside cells. Spatially, the accumulation of the fluorescent intensity, both inside and outside hepatocytes, represented the total amount of resorufin produced.

This process was detected, both temporarily and spatially, by monitoring fluorescent intensity changes over the chip chamber area while the reaction progressed with time. Fig. 3 shows two example snapshots at reaction time zero and $10 \mathrm{~min}$ to compare the fluorescent intensity changes in both cells and the surrounding media. It can be seen clearly that at the beginning of the reaction resorufin production started showing significant fluorescence within the cells only (Fig. 3a). With the increase in resorufin concentration inside the cells, resorufin diffusion across the cell membrane towards the outside became more significant, resulting in a bright fluorescence in the media within the whole chamber (Fig. 3b).

Fig. 4 compares the integrated fluorescent intensities from cells, the surrounding media and the whole circular chamber. It was seen that the integrated fluorescent intensity of all cells was just about $10 \%$ of that from the surrounding media, although the cell area was generally brighter. To obtain the total amount of the resorufin produced during the process, the fluorescent intensity from the whole circular chamber area was used because it represents the analyte in both cells and media, which could be converted to a concentration-dependant calibration model.

To correlate the fluorescent intensity with the resorufin concentrations within the chamber, a calibration method was carried out in which the fluorescent intensity was measured over the whole circular chamber area with a wide range of resorufin concentrations. It was observed that the fluorescent intensity gave a good linear relationship $(\mathrm{Int}=1.7554 \times$ Conc -0.0095$)$ with the resorufin concentration in the range of $0-30 \mu \mathrm{M}$ (Fig. 5). Above that concentration, the fluorescent intensity tended to be lower than predicted, which was thought to be caused by the saturation of the CCD detector. Since the working concentration was normally in the range of a few $\mu \mathrm{M}$ the linear correlation was considered suitable for determining the resorufin concentration in this study. Using the calibration curve the resorufin concentration profile was obtained. Fig. 5 shows a plot of the resorufin concentration as a function of time. It can be seen that the general trend followed the profile of the fluorescent intensity. The blank result is also included for comparison, which indicated no significant increase in fluorescent intensity.
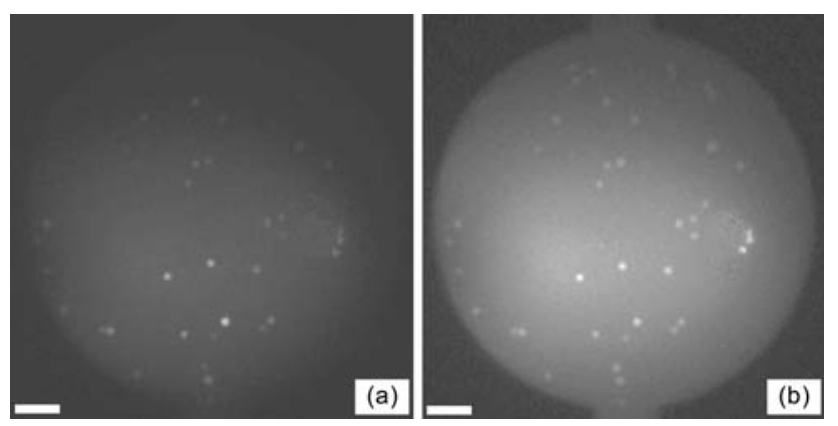

Fig. 3 Fluorescent intensity variations in both cells and media during the reaction of EROD at time zero (a) and $10 \mathrm{~min}$ (b). Scale bar: $100 \mu \mathrm{m}$. (A weak transmission light was applied for a better visibility of the chamber and channel).

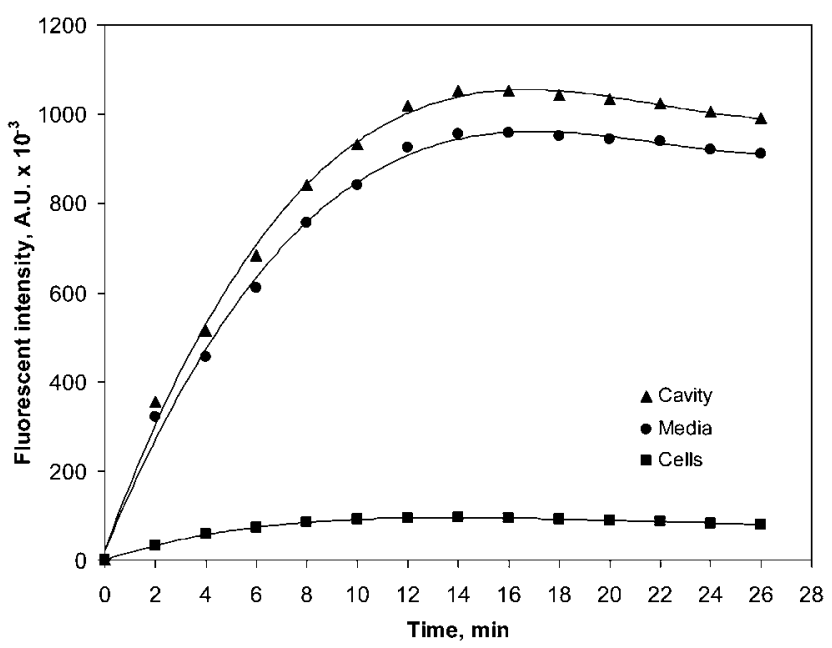

Fig. 4 Integrated fluorescent intensity from areas covering the cells, media or whole circular chamber.

\subsection{Inhibition of EROD activity}

CYP1A enzymes are active in the biotransformation of both endogenous substances and xenobiotics. ${ }^{25}$ They can activate innocuous promutagens into their mutagenic and/or carcinogenic forms through the production of highly reactive epoxide intermediates that can bind potently to DNA and initiate carcinogenesis. ${ }^{26}$ Thus, the examination of the inhibition of CYP1A enzymatic activity is important in the studies of toxicology and drug metabolism, whereas hepatic microsomal EROD activity is an indicator of CYP1A. ${ }^{27}$

In this study, the effect of a model CYP1A inhibitor $\alpha$-NF was examined on the 3-MC-induced rat hepatocytes when various concentrations of $\alpha-\mathrm{NF}$ were added to the test reagent for a range of concentrations $(0-40 \mu \mathrm{M})$. EROD activity was measured using the procedure described above under identical fluidic conditions, and the results are summarised in Fig. 6. It was found that with higher inhibitor concentrations the corresponding fluorescent intensity became lower, as expected. When the concentration of $\alpha$-NF reached $40 \mu \mathrm{M}$, no significant EROD

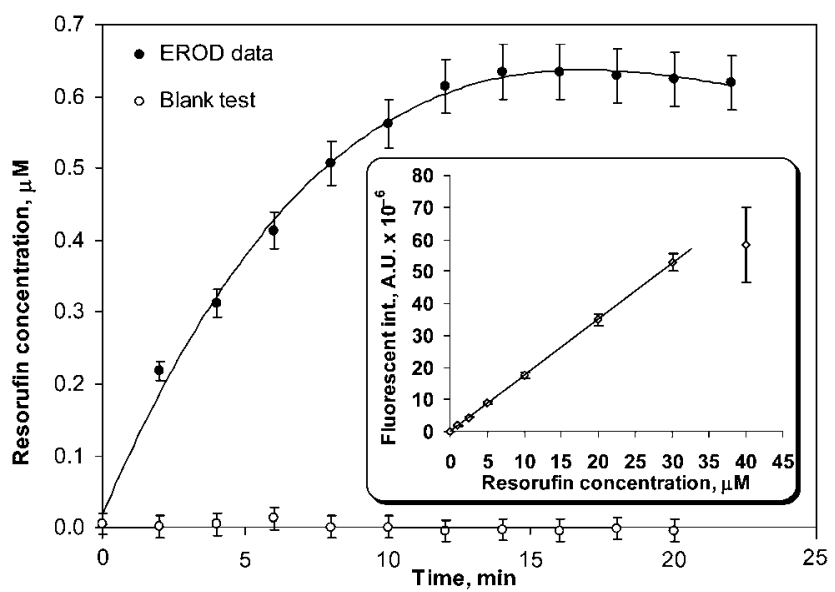

Fig. 5 Resorufin production as a function of time, and (inset) the calibration of fluorescent intensity $v s$. resorufin concentration. 


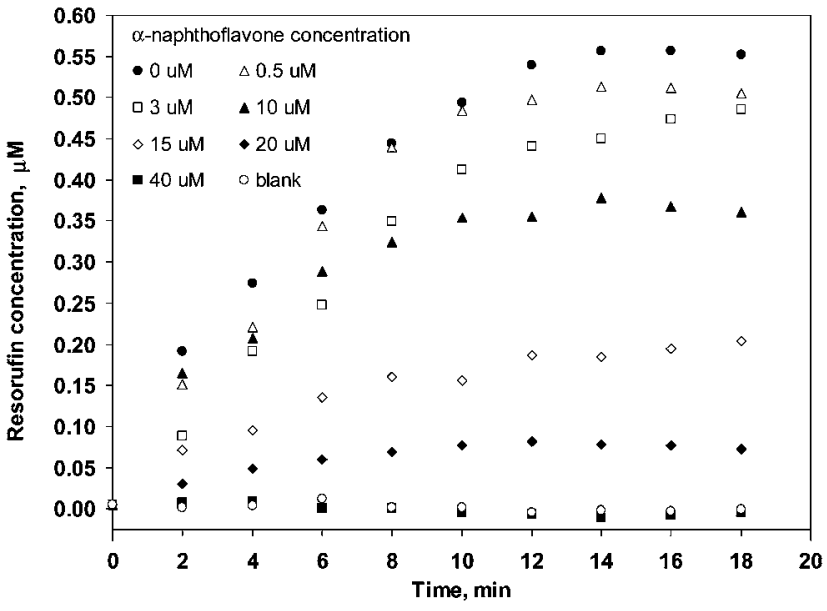

Fig. 6 Resorufin production as a function of time with inhibitor $\alpha-\mathrm{NF}$ at different concentrations.

activity was observed, which was comparable with the result of the blank test. The relationship between the inhibition effect and inhibitor $\alpha-\mathrm{NF}$ concentration is plotted in Fig. 7, where the inhibition effect is expressed as a percentage of the EROD activity remaining.

In biochemical and biomedical studies for dose-response relationships, a four-parameter logistic model has frequently been used to obtain the $\mathrm{IC}_{50}$ value in drug testing $\left(\mathrm{IC}_{50}\right.$ represents the concentration of an inhibitor at which $50 \%$ of the maximal response is observed). ${ }^{28,29}$ This model was employed for fitting the experimental data shown in Fig. 6:

$$
y=\frac{A-D}{1+(x / C)^{B}}+D
$$

where $y$ is the EROD activity remaining as a percentage and $x$ is the inhibitor $\alpha-\mathrm{NF}$ concentration $(\mu \mathrm{M})$. $A$ and $D$ are the upper and lower asymptotes, respectively. $B$ is the slope of the curve (or Hill slope) and $C$ is the central point of the linear portion of the curve, i.e., $\mathrm{IC}_{50}$. By fitting the experimental data to the fourparameter logistic model, the best fit of the four parameters was found to be $98.63,-2.27,3.36$ and 12.98 , respectively.

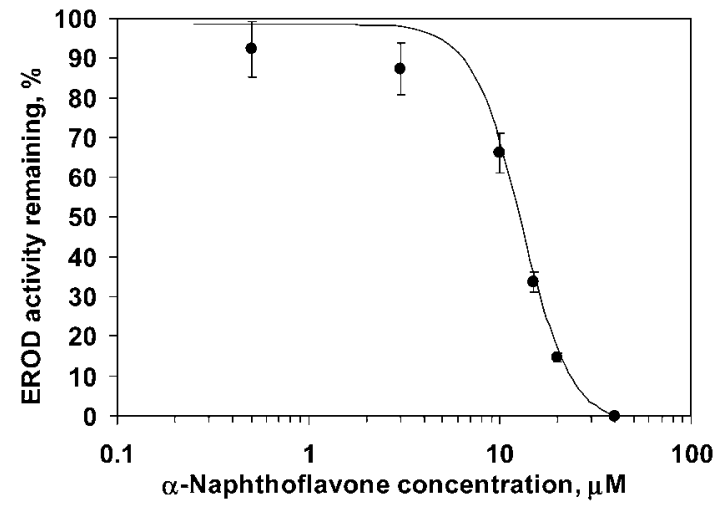

Fig. 7 Effects of various concentrations of inhibitor $\alpha$-NF. The inhibition effect is expressed as percentage of EROD activity remaining.

\section{Conclusions}

A microfluidic-based system was developed for the in situ monitoring of EROD activity for primary rat hepatocytes by measuring the fluorescent intensity of both single cells and their surrounding media. The glass microfluidic chip enabled the cell suspension and test reagent to be introduced in a layer-by-layer flow format, thereby resulting in a short mixing time by diffusion. A calibration showed a good linear relationship between the resorufin concentration up to $30 \mu \mathrm{M}$ and fluorescent intensity. EROD activity was measured with 3-MC-induced hepatocytes, and the inhibition effect of $\alpha-\mathrm{NF}$ was also examined on EROD activity.

\section{Acknowledgements}

Financial support from the UK DTI and UKIERI $\uparrow$ is gratefully acknowledged.

\section{References}

1 M. J. Gomez-Lechon, J. V. Castell and M. T. Donato, Chem.-Biol. Interact., 2007, 168, 30-50.

2 T. Lynch and A. Price, Am. Fam. Physician, 2007, 76, 391-396.

3 H. N. Chaobal and E. D. Kharasch, Clin. Pharmacol. Ther., 2005, 78, 529-539.

4 C. A. Schumann, A. Dorrenhaus, J. Franzke, P. Lampen, P. S. Dittrich, A. Manz and P. H. Roos, Anal. Bioanal. Chem., 2008, 392, 1159-1166.

5 H. L. Tsang, S. C. Wu, C. K. Wong, C. K. Leung, S. Tao and M. H. Wong, Environ. Int., 2009, 35, 1040-1047.

6 M. D. Burke and R. T. Mayer, Drug Metab. Dispos., 1974, 2, 583-588.

7 A. Behrens, K. Schirmer, N. C. Bols and H. Segner, Mar. Environ. Res., 1998, 46, 369-373.

8 G. Zamaratskaia and V. Zlabek, Sensors, 2009, 9, 2134-2147.

9 S. Lindstrom, M. Hammond, H. Brismar, H. Andersson-Svahn and A. Ahmadian, Lab Chip, 2009, 9, 3465-3471.

10 X. Li, X. Xue and P. C. H. Li, Integr. Biol., 2009, 1, 90-98.

11 S.-W. Lee, J. Y. Kang, I.-H. Lee, S.-S. Ryu, S.-M. Kwak, K.-S. Shin, C. Kim, H.-I. Jung and T.-S. Kim, Sens. Actuators, A, 2008, 143, 64 69.

12 L. Charon, A. R. Wheeler and L. Lilge, in Encyclopedia of Micro- and Nanofluidics, ed. D. Q. Li, Springer, Heidelberg, Editon edn, 2008, vol. 3, pp. 1851-1859.

13 O. Hofmann, P. Miller, P. Sullivan, T. S. Jones, J. C. deMello, D. D. C. Bradley and A. J. deMello, Sens. Actuators, B, 2005, 106, 878-884.

14 A. L. Paguirigan and D. J. Beebe, BioEssays, 2008, 30, 811-821.

15 X. L. Zhang, H. B. Yin, J. M. Cooper and S. J. Haswell, Anal. Bioanal. Chem., 2008, 390, 833-840.

16 P. Chao, T. Maguire, E. Novik, K. C. Cheng and M. L. Yarmush, Biochem. Pharmacol., 2009, 78, 625-632.

17 X. L. Zhang, H. B. Yin, J. M. Cooper and S. J. Haswell, Electrophoresis, 2006, 27, 5093-5100.

18 T. McCreedy, Anal. Chim. Acta, 2001, 427, 39-43.

19 K. Behnia, S. Bhatia, N. Jastromb, U. Balis, S. Sullivan, M. Yarmush and M. Toner, Tissue Eng., 2000, 6, 467-479.

20 A. Fick, Ann. Phys. Chem., 1855, 94, 59-86.

21 E. A. Schilling, A. E. Kamholz and P. Yager, Anal. Chem., 2002, 74, 1798-1804.

† This document is an output partially from the UKIERI (UK-India Education and Research Initiative) project funded by the Department for Innovation, Universities and Skills (DIUS), the FCO, British Council, Department of Science and Technology, Government of India, The Scottish Government, Department of Learning, Northern Ireland, Welsh Assembly, GSK, BP, SHELL and BAE for the benefit of the UK and Indian Higher Education Sector. The views expressed are not necessarily those of the funding bodies. 
22 S. Lindström, R. Larsson and H. A. Svahn, Electrophoresis, 2008, 29, 1219-1227.

23 M. T. Donato, E. Herrero, M. J. Gomezlechon and J. V. Castell, Toxicol. Vitro, 1993, 7, 481-485.

24 D. D. Vakharia, N. Liu, R. Pause, M. Fasco, E. Bessette, Q. Y. Zhang and L. S. Kaminsky, Drug Metab. Dispos., 2001, 29, 999-1006.

25 D. L. Anger, M. A. Petre and D. J. Crankshaw, Br. J. Pharmacol., 2005, 145, 926-933.
26 F. J. Gonzalez and H. V. Gelboin, Drug Metab. Rev., 1994, 26, 165183.

27 P. V. Nerurkar, S. S. Park, P. E. Thomas, R. W. Nims and R. A. Lubet, Biochem. Pharmacol., 1993, 46, 933-943.

28 O. Ugur and H. O. Onaran, Biochem. J., 1997, 323, 765776.

29 Y. I. Yagi, K. Abe, K. Ikebukuro and K. Sode, Biochemistry, 2009, 48, 10255-10266. 